\title{
EDITORIAL
}

\section{INDUSTRIA DE LA CONSTRUCCIÓN: ¿FRAGMENTADA O INTEGRADA?}

\section{CONSTRUCTION INDUSTRY: FRAGMENTED OR INTEGRATED?}

DOI: https://doi.org/10.51372/gacetatecnica231.1

Últimamente se encuentran términos dentro en la industria AEC (Arquitectura, Ingeniería y construcción por sus siglas en inglés), como planificación colaborativa, diseño concurrente, equipos integrados, planificación e integración temprana. Estos responden a metodologías no tradicionales que traen consigo altos beneficios de ahorros de tiempos, costos, progresos en la productividad y calidad, mejoras notables en la constructabilidad, así como en la fluidez de información $y$ comunicación del proyecto

La industria AEC, sin embargo, es conocida por tener gran cantidad de disciplinas asociadas que trabajan de forma disgregada, por el largo ciclo de vida de los proyectos, la alta rotación de personal y alta demanda de mano de obra, características que hacen que se perciba como una industria fragmentada, pudiéndose visualizar en tres dimensiones. La primera es la temporal, no toda la información y comunicación fluye en el tiempo, desde las primeras fases hasta las últimas, lo que se realiza en el diseño, no necesariamente se realiza de esa forma en la ejecución. La segunda es espacial, significa que, en un mismo momento, los equipos trabajan de forma separada pudiendo presentarse inconsistencias en sus resultados preliminares y al momento de coordinar distintas especialidades. La tercera es transversal, entre proyectos, referido a que cada proyecto de una misma empresa trabaja a su forma, sin aprender de otros proyectos simultáneos o pasados.

Aunado a esto, los contratos que son empleados en la industria AEC son tradicionalmente de diseño, licitación y construcción (DBB-Design-Bid-Build), es decir, que luego que el equipo de diseño ha culminado totalmente el proyecto y sus entregables, se realiza la licitación o concurso para determinar quién ejecutará la obra. Esta manera de trabajar de forma tan segmentada ha traído históricamente consecuencias de retrabajos, pérdidas de tiempo, gran cantidad de solicitudes de información del proyecto, sobrecostos y sobretiempos.

En otros países, como Reino Unido, por ejemplo, se utiliza en el sector público de forma predeterminada el Design-Build o Diseño-Construcción, en el cual, la relación entre los equipos de diseño y construcción es un poco más integrada. Igualmente, se está haciendo más fuerte en países desarrollados, el uso del Integrated Project Delivery (IPD) que algunos asocian con Lean Project Delivery (LPD) por la incorporación de herramientas de Lean Management en el proceso. Tanto el IPD como $L P D$, añaden cambios interesantes en varios aspectos del proyecto como la 
incorporación temprana del constructor o participantes claves; respeto y confianza entre contratante, contratista, diseñadores, consultores, subcontratistas e incluso proveedores; innovación y tomas de decisiones en forma colaborativa; compartir riesgos y recompensas; comunicación abierta; planificación y diseño intensivo; definición temprana de objetivos del proyecto, y por último, uso de tecnología apropiada.

Las barreras para implementar en los proyectos un sistema integrado son altas, se tienen grandes desafíos en la industria. Como investigadores, consultores, profesores, profesionales y estudiantes del área tenemos el deber de convertirnos en agentes de cambio, buscando estrategias que permitan mejorar los procesos hacia la transparencia y confianza en las comunicaciones, trabajando con un fin común desde diferentes perspectivas y disciplinas para beneficio del proyecto y las comunidades que se verán favorecidas. Formándonos en tecnologías de punta (BIM-Building Information Modeling, modelos $n D$, Construcción y Diseño virtual, gemelos digitales) para alcanzar mejores desempeños y generar más valor para todos los actores involucrados del proceso y producto de los proyectos. Aún nos falta mucho que aprender, innovar, implementar, probar, investigar, mejorar, alcanzar... ¿estamos dispuestos?

Dra. Zulay Giménez Palavicini

Pontificia Universidad Católica de Chile

Profesora Escuela de Construcción Civil zmgimenez@uc.cl 